\title{
Development of a Low-Cost Bio-Inspired Swimming Robot (SRob) with IoT
}

\author{
Mohd Aliff ${ }^{1}$, Ahmad Raziq Mirza ${ }^{2}$, Mohd Ismail $^{3}$ \\ Instrumentation and Control Engineering \\ Malaysian Institute of Industrial Technology \\ Universiti Kuala Lumpur \\ Johor, Malaysia
}

\author{
Nor Samsiah ${ }^{4}$ \\ Center for Artificial Intelligence Technology (CAIT) \\ Faculty of Information Science and Technology \\ Universiti Kebangsaan Malaysia \\ Selangor, Malaysia
}

\begin{abstract}
Now-a-days, exploring underwater is a difficult activity to do and requires specialized equipment to explore it. Many studies so far have proven that bio-inspired robotic fish such as stingray robots have many advantages for use as underwater exploration. One of them is the manta ray which can show excellent swimming ability by flapping the pectoral fins with large amplitude. By studying the movement behavior of genus Mobula, the development of biomimetic robots has grown exponentially in recent years. But this technology requires expensive development costs, and the prototypes produced are heavy. Therefore, the development of low-cost bio-inspired Swimming Robot (SRob) using embedded controller with internet of things (IOT) is proposed and presented in this paper. SRob is designed with a small size and lightweight compared to other conventional swimming robots and is well equipped with 6 servo motors, ADXL335 accelerometer 3-axis, 2 Lipo batteries 7.4V, ESP01 Wi-Fi module and Arduino Mega. The RemoteXY app that works like a remote control will be connected to the Arduino Mega using the ESP01 Wi-Fi module to control servo motors and obtain readings of the sensors. Based on the experimental results, the servo motor used to produce flapping motion can be controlled precisely while producing a large amplitude of motion. In addition, the position control for the compact SRob can be realized and determined correctly while swimming in the water.
\end{abstract}

Keywords-Stingray robot; angle of flapping motion; remote control; position control; compact SRob

\section{INTRODUCTION}

Numerous studies have demonstrated that robots are particularly effective in medical [1], rehabilitation [2-7], rescue operations [8-10], automobiles, and manufacturing [11-12]. Robotics has been used in a variety of industries for many years and has provided humans with numerous benefits. Furthermore, as the internet has grown in popularity, more intelligent devices and systems have emerged. The concept of internet or web-based controls for robots and the home environment is still in its early stages. The Internet offers lowcost communication routes and can be used for telephony. Many issues still need to be addressed before a viable realworld application can be realized. Researchers across the world are interested in using the internet to control robots as part of the Fourth Industrial Revolution (4IR).
Manta rays are the world's largest and most intelligent rays. The swimming capabilities of the manta ray has recently attracted the interest of researchers around the world and focusing on its movement performance, maneuverability, and stability [13]. Relevant research has been conducted based on this movement pattern and prototypes such as fish robots have been developed. However, the previous research on this swimming robot only focuses on the design and propulsion performance in addition to its expensive production cost and its large and heavy size.

Currently, many research studies have developed underwater vehicles fitted with propellers for use in challenging water environments. While it is easy to operate, there are some drawbacks on its movement mode such as loud noise, large scale, poor performance and even low speed maneuvers. There are also some underwater vehicles such as Autonomous Underwater Vehicles (AUV) and Unmanned Underwater Vehicle (UUV) that rely heavily on the design of manta rays and are able to glide in water without human control. Both robotic vehicles are heavily equipped with various types of sensors and cameras to be able to collect various types of data while used in rescue operations or exploration in the water to find out the environmental conditions.

Bio-inspired robotics provides biologists the equipment to study animal behavior and test beds for research and evaluation of biological algorithms for potential engineering applications [14]. Various studies involving animals including invertebrates and vertebrates have been conducted for different purposes. One of the research projects involving bio-inspired robots is used to answer simple biological questions or build classical mechatronic structures [15]. The development of swimming robot is particularly promising, not only as a testbed for learning how fish swim, but also for different underwater applications such as underwater discovery, patrol, and aquatic surveillance. Despite their appearance, current robotic fish are still substantially slower and less durable than their biological counterparts, necessitating further research into swimming mechanisms and control methods. Rajiforms are a type of underwater animal that have long wavy fins and they have a variety of swimming methods. The ray species that demonstrate this two-fin swimming method are manta rays with swinging flaps and stingrays with wavy movements. Rays 
can make large deformations on the fins because their bodies have no bone structure. In particular, manta rays can produce large amplitudes of deformation with fast duration and large thrust [16].

The main objectives of this project are to develop the manta ray swimming robot (SRob) using Arduino and WiFi module, to control and monitor manta ray swimming robot using RemoteXY application and ADXL335 accelerometer and to evaluate the movement of manta ray swimming robot based on angle of servo motor. The scope of this project focuses on control system using embedded controller along with IoT for manta ray robot prototype while solving the problem of conventional swimming robots by developing lightweight prototype at low cost.

\section{LITERATURE REVIEW}

Although bionic manta ray research has progressed significantly in recent years, robot mobility still differs significantly from that of genuine manta rays. To begin with, most existing bionic manta rays use an oscillating propulsion mode, and the mechanical construction is typically simplified. A prototype's skeleton is usually built of stiff material. As a result, the majority of prototypes are rigid. Second, the bionic prototype's control algorithm cannot quite match the genuine movement of manta rays, especially during the transition between motor patterns. As a result, to improve control performance, a more robust and reasonable control algorithm is necessary. The bionic manta ray robot with flexible pectoral fin was proposed in response to the mechanical structure and motion control algorithm shortcomings of previous bionic manta rays [17]. The longitudinal structure of the pectoral fins was created using 3D printing technology and joined by flexible beams and metal wires. As a result, these pectoral fins' geometrical form was more akin to that of actual manta rays. CPG was used to recreate the mobility pattern of manta rays based on observations of their periodic movement. However, the prototype that was invented was still quite huge and heavy. The bionic manta ray robot is $48.5 \mathrm{~cm}$ long, $70.0 \mathrm{~cm}$ wide, and $6.0 \mathrm{~cm}$ tall. It weighs $6 \mathrm{~kg}$ and has a maximum speed of 0.4 $\mathrm{m} / \mathrm{s}$ with a flapping frequency of $2.5 \mathrm{~Hz}$.

Alvarado et al. using a new method that can provide an advantage in its robotic stingrays, which are made of a soft material and only require one actuator per fin [18-21]. Chew et al. demonstrated a Robot Manta Ray prototype in which each fin's oscillatory motion is controlled by a single servo motor [22]. The fin is constructed with a leading-edge structure/spare that supports a flexible fin film material consisting of PVC film of uniform thickness, with a flapping fin frequency of $1.1 \mathrm{~Hz}$ and a maximum speed of $1.783 \mathrm{BL} / \mathrm{s}$. The replaced servomechanism was clamped above the water while the fin was flapping near the water surface to measure thrust created by a single fin. However, the effect of the water surface and the movement of the separated actuator may not be consistent with the robot manta ray, and the thrust performance of a Robo manta ray has not been evaluated using this measurement setup.

Batoids achieve extraordinary mobility and propulsive performance thanks to their huge pectoral fins. The robot is made to move in an undulatory rajiform pattern. Because of its full autonomy, extended battery life, and wireless recharging capabilities, the design is under-actuated, simple, and sturdy, making it ideal for propulsive performance investigations. The robot has an overall length of $180 \mathrm{~mm}$ with a top speed of 0.93 body length per second [23]. Based on the experimental results, both thrust and side forces are found to rise as flapping frequency and amplitude increase, but frequency effects are more obvious. The proposed robot designs greatest swimming speed is close to $1 \mathrm{BL} / \mathrm{s}$. For the identical input parameters, side forces were greater than thrust forces for the geometry and swimming mode matched by the design [23].

\section{MethodOLOGY}

Fig. 1 shows the schematic wiring diagram for controlling SRob. SRob has been equipped with various components, including 6 servo motors, an ADXL335 3-axis accelerometer, 2 Lipo batteries, an ESP01 WiFi module, and an Arduino Mega, to achieve the desired movement and speed. On the hardware development side, AutoCAD 2017 was used to create the 3D design, while Fritzing was used to create the wire diagrams for the control system. The servo motor consists of three colors of wire that the black wire connects to the GND, the white or yellow wire connects to the pin and the red wire connects to the power supply. The servo motor requires a voltage of around $4.8 \mathrm{~V}-6.0 \mathrm{~V}$. The speed of the servo motor stays $0.14 \mathrm{sec} / 60$ degrees for $4.8 \mathrm{~V}$ and $0.12 \mathrm{sec} / 60$ degrees for 6.0V. ADXL335 is an accelerometer sensor that works based on the principle of Piezoelectric effect. Each time the sensor is tilted, the ball will move in that direction due to the force of gravity. The wall is made of Piezoelectric elements and if every time the ball touches the wall, an electric current will be generated which will be interpreted in the form of a value in any 3D space. In addition, the ADXL335 will provide acceleration values on three axes and will provide three output values. The bandwidth of this sensor is adjustable as it has a single capacitor per axis. Analog interfaces are made to communicate with other devices such as Arduino. This accelerometer sensor requires a voltage of around $1.8 \mathrm{~V}$ to $3.6 \mathrm{~V}$.

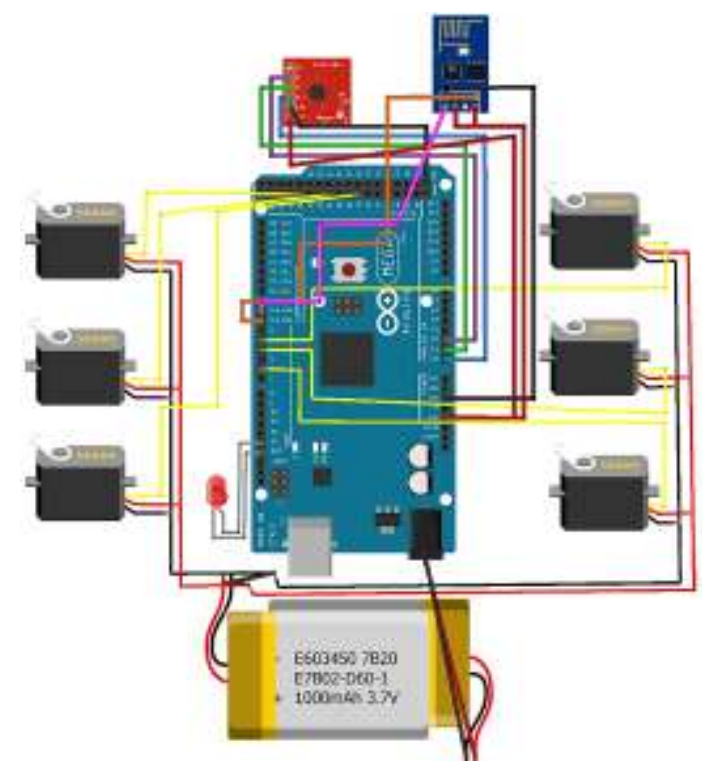

Fig. 1. Schematic Wiring Diagram. 
Serving as the brain for the SRob, the Arduino Mega 2560 is one of the microcontroller boards used to control the movement of the swimming robot. This microcontroller has 54 digital input and output pins where 15 pins can be used as PWM output. The ESP01 Wi-Fi module is a complete and standalone solution for Wi-Fi networks that can carry application services and connect SRob to the internet. This WiFi module is very important making the SRob can be controlled remotely and wirelessly. This feature makes it easier for the operator to control the movement of the SRob effectively without having to be in a hazardous environment. The ESP8266 high frequency clock is used to drive two Tx and Rx mixers provided by the internal oscillator and the external oscillator. The frequency of the floating crystal is $26 \mathrm{MHz}$ to $52 \mathrm{MHz}$. The ESP01 Wi-Fi module requires a $3.3 \mathrm{~V}$ power supply and an ambient temperature of 25 degrees Celsius. Then, from the internet cloud, SRob will be connected directly to the RemoteXY application which serves as a remote control to navigate SRob from a smartphone. The control process starts from the power source which is the Lipo battery which will activate the microcontroller, sensor, and actuator. Then, the accelerometer will calculate the exact body force and angular rate so that the exact position of the SRob can be determined. Finally, the servo motor will be activated and will operate according to the instructions from the remote control whether moving forward, turning right, left, or turning 360 degrees.

\section{CONTROL SYSTEM}

Fig. 2 shows the 3D design of the SRob. Each side of the main body is fitted with three servo motors that are used to generate thrust. These three servo motors have been set up to have different delays from each rotation and can produce large wave motion. Thus, this will allow the SRob to move forward or change direction turning to the right or left in a short time. The main body of the SRob is made of durable acrylic and has a specific weight that is suitable to make the SRob sink and float in water. This main body houses all the components including the Arduino Mega, ADXL335 accelerometer-3axis, Lipo battery, and ESP01 WiFi module. The pectoral fin material must be flexible and waterproof so that swimming movements can be produced smoothly. The height of the robot manta ray is $6 \mathrm{~cm}$, width and length are $80 \mathrm{~cm}$ and $30 \mathrm{~cm}$, respectively.

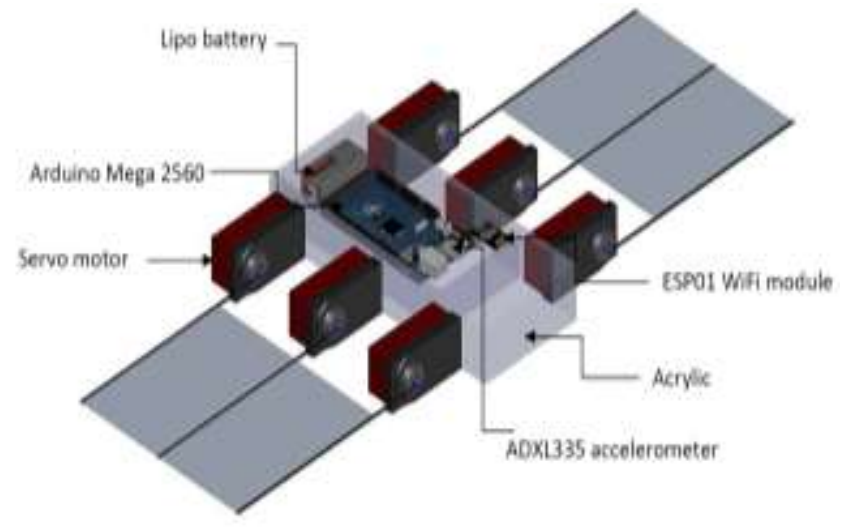

Fig. 2. 3D Design of the SRob.
Fig. 3 shows the flowchart of the SRob control system. After connecting all the parts together, the RemoteXY app must be connected to the Arduino Mega using WiFi to control all the servo motors. After that, the SRob can be activated on the smartphone, and at the same time, the accelerometer sensor will measure and calculate the axial position of the SRob. This will help the operator to know the initial position of the SRob before any operation is carried out. To control the movement of the SRob, there are five different types of modes have been set. The first mode is a command for the robot to stop. When the $A$ button is pressed on the remote control using the RemoteXY application on the smartphone, all servo motors will turn off. The second mode is to move forward. When button $B$ is pressed, all servo motors will be turned on and thrust will be generated making the SRob move forward. The third mode is to move forward to the right. When the $C$ button is pressed, all servo motors on the right will be turned off causing only the left side to produce movement. The fourth mode is to move forward to the left. The same command as to the right is used but only the servo motor on the left will be turned off while the servo motor on the right will be turned on. Lastly is the fifth mode which is a $360^{\circ}$ rotation round. When the $E$ button is pressed, each side of the servo motor will produce the opposite direction so that the SRob can rotate up to $360^{\circ}$.

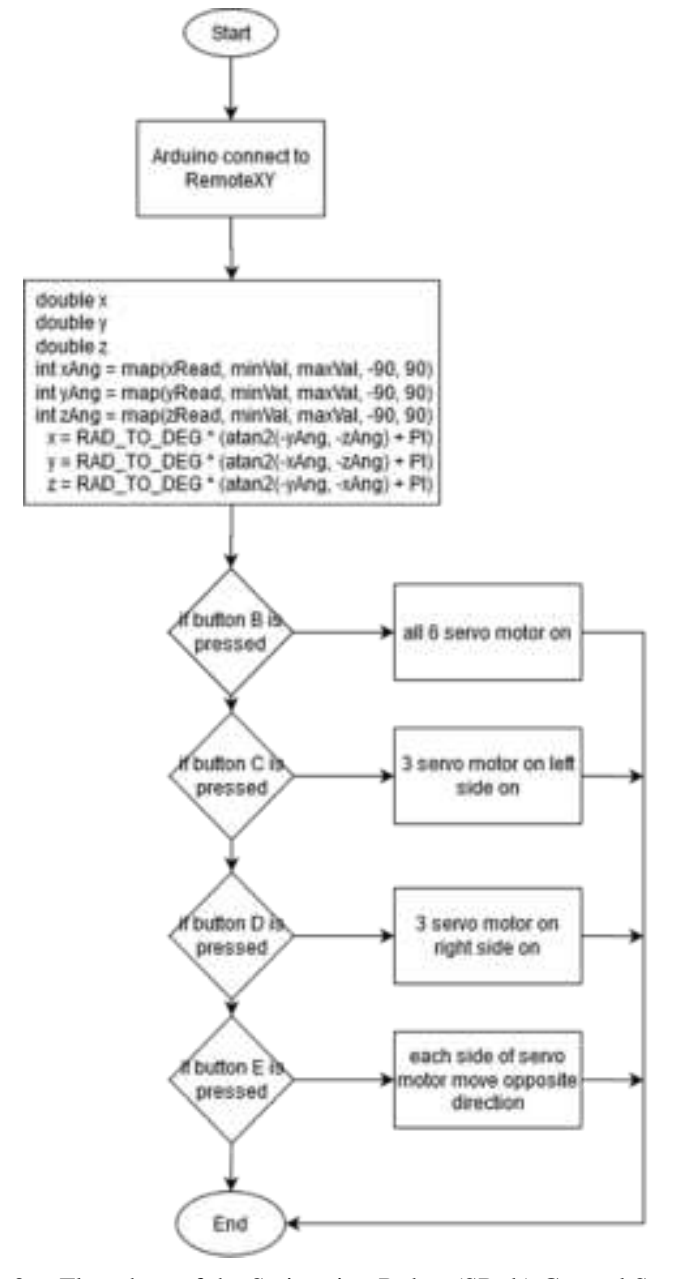

Fig. 3. Flowchart of the Swimming Robot (SRob) Control System. 


\section{RESUlT AND DiscUSSION}

Fig. 4 shows the speed of the SRob against time. From this graph, SRob takes 10 seconds to move forward $2 \mathrm{~m}$ in the pool. The maximum speed that can be reached by the prototype is 20 $\mathrm{m} / \mathrm{s}$ and takes 1 second to reach the maximum speed. Based on the graph, the data shows that the time increases when the speed reaches 1 second and remains the same up to 10 seconds. Fig. 5 shows the flapping motion angle relative to the time for moving forward produced by 6 servo motors. The angle for the servo motor to operate is between $70^{\circ}$ to $110^{\circ}$, while the difference value between the initial angle and the final angle is $40^{\circ}$. According to the findings of the research, improper angles result in imbalanced speed and movement. As a result, a 40degree angle was set on the microcontroller to achieve maximum speed and smooth movement. Based on this graph it is also found that the servo motor takes 0.75 seconds to complete one cycle.

Fig. 6 shows the flapping motion angle relative to the time to move to the right. The three straight lines in this graph indicate the 3 servo motors on the right that are not energized while the three sinusoidal lines are the three servo motors that are energized on the left side of the SRob. When the $C$ button is pressed, servo motor 1,2 and 3 on the left will be actuated while the other servo motors are not activated. The graph also shows that a servo motor takes 0.45 seconds to complete one cycle compared to 0.75 seconds found in Fig. 5. This function is important to ensure that the SRob can turn right or left faster. Fig. 7 shows the flapping motion angle relative to the time to move to the left. This graph, like the method of turning to the right, includes three straight lines indicating that the three servo motors on the left are not powered and three sinusoidal lines indicating that the three servo motors on the right are energized. When the $D$ button is pressed, the servo motor 4,5 and 6 on the right will be actuated while the other servo motors are not energized.

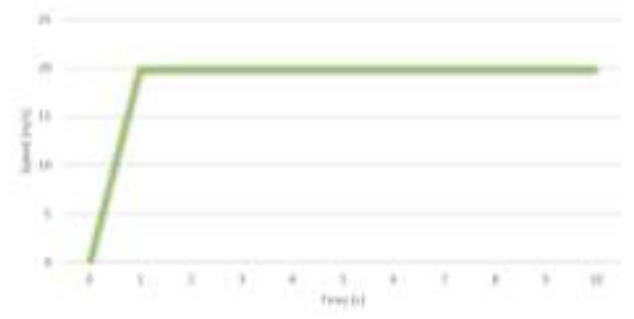

Fig. 4. Speed of the SRob vs Time.

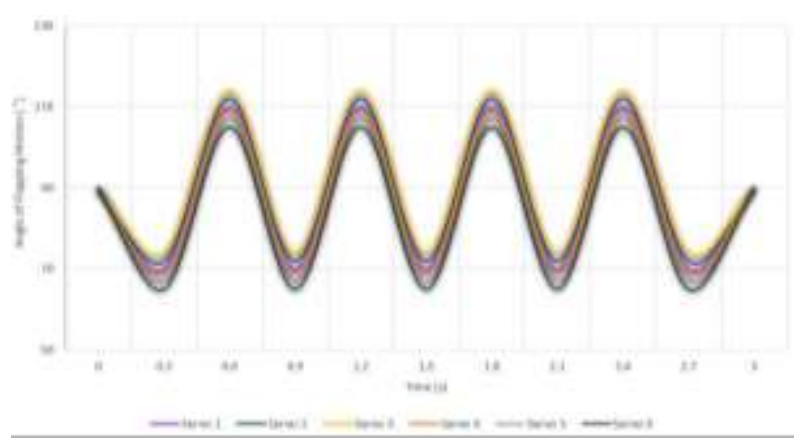

Fig. 5. Angle of Flapping Motion vs Time for Moving Forward.

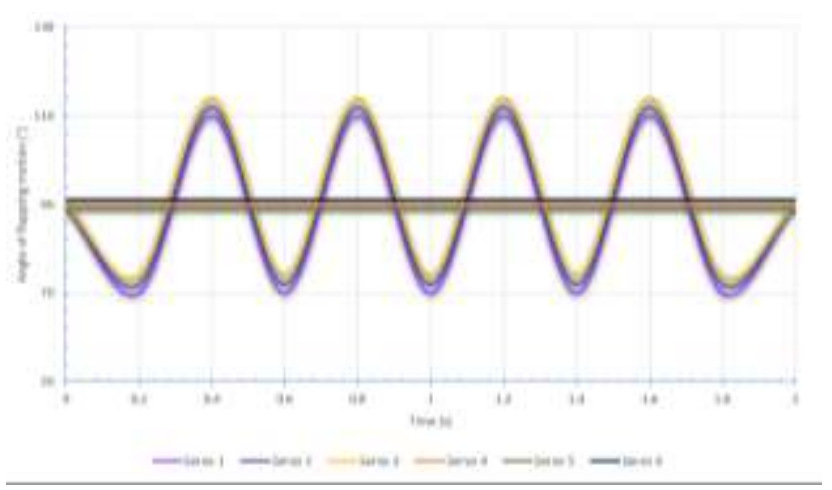

Fig. 6. Flapping Motion Angle Relative to the Time to Move to the Right.

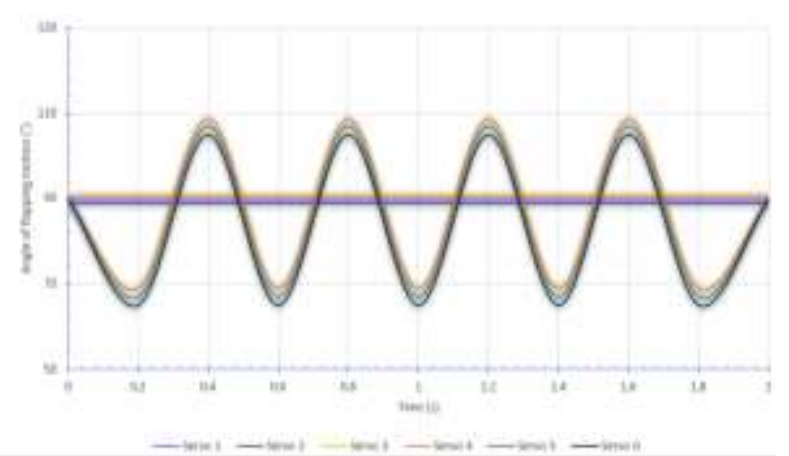

Fig. 7. Flapping Motion Angle Relative to the Time to Move to the Left.

Fig. 8 shows the flapping motion angle relative to the time to rotate $360^{\circ}$. There are two sinusoidal graphs representing each servo motor for the SRob. To ensure that the SRob can rotate $360^{\circ}$, the initial angle of the three servo motors on the right is set to $110^{\circ}$, while the initial angle of the three servo motors on the left is set to $70^{\circ}$. When the $E$ button is pressed, the three servo motors on the right will undulate from $70^{\circ}$ to $110^{\circ}$ while the three servo motors on the left will undulate from $110^{\circ}$ to $70^{\circ}$. As a result, by setting the angle accurately, it will produce two sinusoidal graphs, and the SRob will be able to rotate at a fast and effective rate. Data for the position of the SRob were taken using an ADXL335 accelerometer sensor placed on the main body on the flat surface. Data is collected when the SRob is connected to WiFi using the ESP01 WiFi module. Due to the water vibration caused by the servo motor, the data for the $\mathrm{x}$-axis and $\mathrm{y}$-axis showed tiny difference values during the testing, whereas the $\mathrm{z}$-axis had large values.

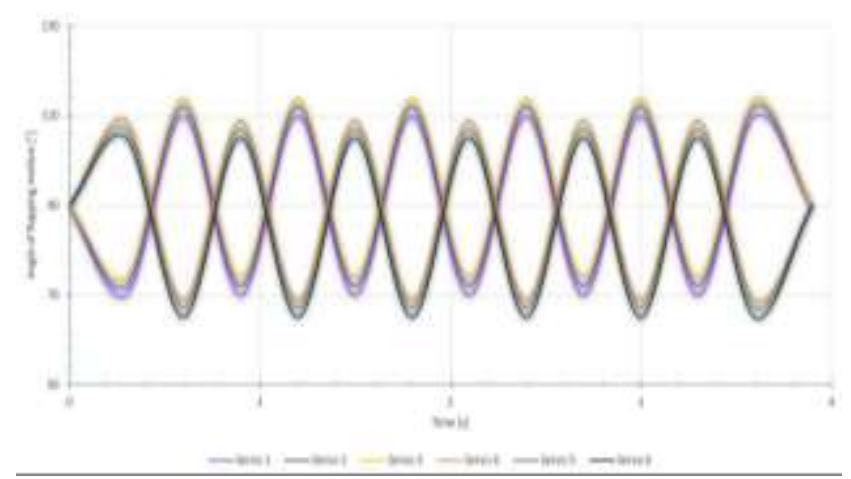

Fig. 8. Flapping Motion Angle Relative to the Time to Rotate $360^{\circ}$. 


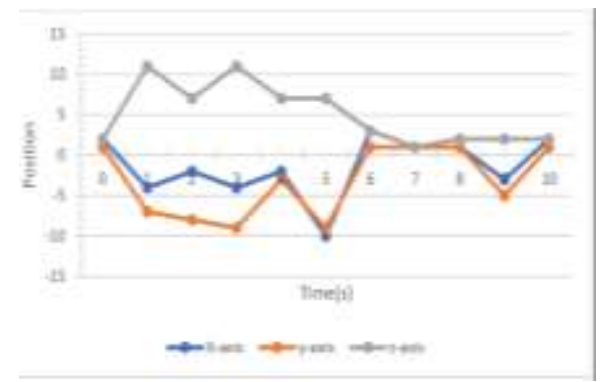

(a) Moving Forward.

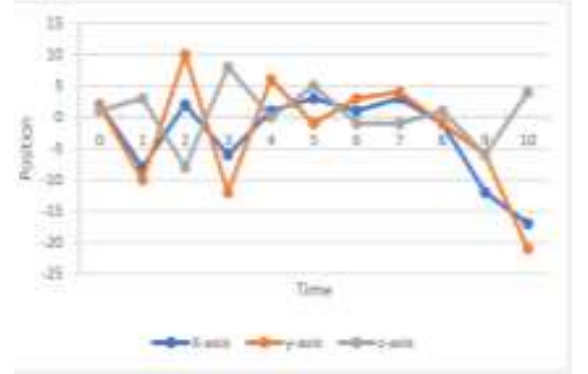

(b) Turning to Right.

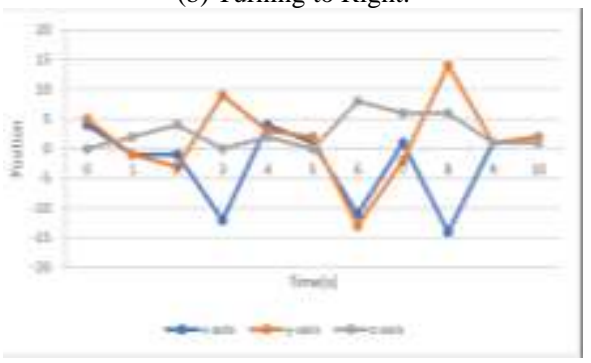

(c) Turning to Left.

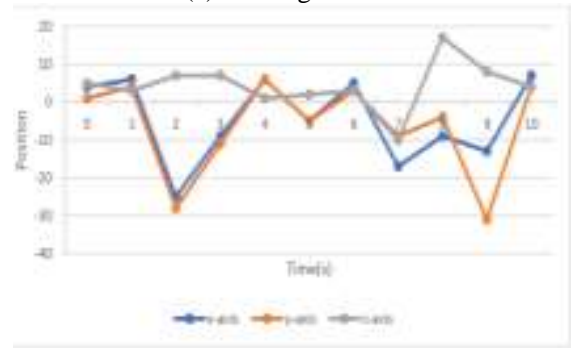

(d) Rotate $360^{\circ}$.

Fig. 9. SRob Position Graphs for $\mathrm{x}$-axis, $\mathrm{y}$-axis, and $\mathrm{z}$-axis vs Time.

Fig. 9 shows the SRob position graphs for $\mathrm{x}$-axis, $\mathrm{y}$-axis, and z-axis vs time for (a) moving forward, (b) turning to right, (c) turning to left, and (d) rotate $360^{\circ}$. For the forward motion test, from 0 seconds to 6 seconds, there was a very large degree difference between the 3 axes. At 10 seconds, the positions of the SRob on the x-axis, y-axis and z-axis were $2^{\circ}, 1^{\circ}$ and $2^{\circ}$, respectively. As the SRob moves to the right, from the 8th to the 10th second, there is a very large difference of degree between the three axes. At 10 seconds, the positions of SRob on the x-axis, y-axis and z-axis were at $17^{\circ}, 21^{\circ}$ and $4^{\circ}$, respectively.

Then, when the SRob moves to the left, starting at the 2nd to 9th second, there is a large degree of difference between the three axes. At 10 seconds, the positions of SRob on the $\mathrm{x}$-axis, $\mathrm{y}$-axis and $\mathrm{z}$-axis were at $1^{\circ}, 2^{\circ}$ and $1^{\circ}$, respectively. Finally, for a $360^{\circ}$ rotation, from the 1 st second to the 4 th second and from the 7 th second to the 10th second, there is a very large degree of difference between the three axes. At 10 seconds, the positions of SRob on the x-axis, y-axis and z-axis were at $7^{\circ}, 4^{\circ}$ and $4^{\circ}$, respectively. From the observations during the experiments performed, due to the water vibration caused by the servo motors, the data for the $\mathrm{x}$-axis and $\mathrm{y}$-axis show small difference values during the testing, whereas the $\mathrm{z}$-axis has large difference values.

\section{CONCLUSION}

The development of a low-cost bio-inspired swimming robot (SRob) with IoT can be summarized as follows. The first objective of this study is to develop the SRob swimming robot using Arduino and $\mathrm{WiFi}$ modules. The inexpensive and compact prototype has been successfully developed using a durable acrylic material and equipped with several essential components such as an Arduino Mega, six servo motors, a $\mathrm{WiFi}$ module, and an accelerometer sensor. Then, to control and monitor the SRob, the RemoteXY app was used on the smartphone as a remote control for the SRob. With this IoT feature, operator can control the SRob easily without having to be in a dangerous area. From the experimental results, it can be concluded that the $\mathrm{z}$-axis has a small difference value of only $4^{\circ}$ compared to the $\mathrm{x}$-axis and $\mathrm{y}$-axis, which have a big degree difference of $17^{\circ}$ and $21^{\circ}$, respectively. By using an accelerometer sensor, the position and condition of the SRob can be determined effectively. Finally, the rotational angle range of the servo motor for fluctuate is between $70^{\circ}$ and $110^{\circ}$ and the maximum speed of the SRob that can be achieved is 20 $\mathrm{m} / \mathrm{s}$. The soft fins' material characterization and design will be the subject of future research. Maneuverability will also be examined further. Furthermore, multiple navigation algorithms will be used to further investigate autonomy.

\section{ACKNOWLEDGMENT}

The authors gratefully acknowledge to the Ministry of Higher Education (MoHE) Malaysia for financial supports given under the Fundamental Research Grant Scheme (FRGS/1/2019/TK04/UNIKL/02/11).

\section{REFERENCES}

[1] S. Jeelani, A. Dany, B. Anand, S. Vandana, T. Maheswaran, and E. Rajkumar, "Robotics and medicine: A scientific rainbow in hospital," Journal of pharmacy \& bioallied sciences, 2015, 7(Suppl 2), S381-S383.

[2] M. Aliff, F. Danieal, M. F. Mohamed, A. 'Athif, T. Akagi, and N. Samsiah, "Development of Flexible Pneumatic Rehabilitation Actuator for Knee Injury," TEST Engineering \& Management, 83, 2020, pp. $12849-12855$.

[3] H. Obayashi, T. Akagi, S. Dohta, W. Kobayashi, Y. Matsui, S. Shimooka, T. Shinohara, and M. Aliff, "Development of Portable Rehabilitation Device Driven by Low-Cost Servo Valve Using Tap Water," International Journal of Mechanical Engineering and Robotics Research, 9(3), 2020, pp. $353-359$.

[4] M. Aliff, M. A. Dinie, I. Yusof, and N. Samsiah, "Development of Smart Glove Rehabilitation Device (SGRD) for Parkinson's Disease," International Journal of Innovative Technology and Exploring Engineering (IJITEE), 9(2), 2019, pp. 4512 - 4518.

[5] M. Aliff and N. Samsiah Sani, "Robot Arm Analysis based on Master Device Pneumatic Actuators," International Journal of Advanced Computer Science and Applications (IJACSA), 10(8), 2019, pp. 199 204. 
[6] M. Aliff, S. Dohta, and T. Akagi, "Control and analysis of simplestructured robot arm using flexible pneumatic cylinders," International Journal of Advanced and Applied Sciences, 4(12), 2017, pp. 151-157.

[7] M. Aliff, S. Dohta, and T. Akagi, "Simple Trajectory Control Method of Robot Arm Using Flexible Pneumatic Cylinders," Journal of Robotics and Mechatronics, 27 (6), 2015, pp. 698-705.

[8] M. Aliff, I. S. Amry, M. I. Yusof, A. Zainal, A. Rohanim, and N. S. Sani, "Development of Smart Rescue Robot with Image Processing (iROB-IP)," International Journal of Electrical Engineering and Technology, 11(9), 2020, pp. 08-19.

[9] M. Aliff, N. Firdaus, N. Rosli, M. I. Yusof, N. Samsiah, and S. Effendy, "Remotely Operated Unmanned Underwater Vehicle for Inspection," International Journal of Innovative Technology and Exploring Engineering (IJITEE), 9(2), 2019, pp. 4644 - 4649.

[10] M. Aliff, N. S. Sani, M. I. Yusof, and A. Zainal, "Development of Fire Fighting Robot (QRob)," International Journal of Advanced Computer Science and Applications (IJACSA), 10(1), 2019, pp. $142-147$.

[11] C. P. Day, "Robotics in Industry-Their Role in Intelligent Manufacturing," Engineering, 4(4), pp. 440-445.

[12] M. Aliff, A. H. Hamdi, I. Yusof, and N. Samsiah, "Development of Control System for Dual NC Machine with Single 6-Axis Robot," International Journal of Innovative Technology and Exploring Engineering (IJITEE), 9(2), 2019, pp. 4505 - 4511.

[13] G. Li, Y. Deng, O. L. Osen, S. Bi, and H. Zhang, "A bio-inspired swimming robot for marine aquaculture applications: From conceptdesign to simulation," OCEANS 2016 - Shanghai, 2016, pp. 1-7.

[14] Q. Shi, H. Ishii, Y. Sugahara, A. Takanishi, Q. Huang, and T. Fukuda, "Design and Control of a Biomimetic Robotic Rat for Interaction With Laboratory Rats," IEEE/ASME Transactions on Mechatronics, vol. 20, no. 4, 2015, pp. 1832-1842.

[15] J. Yu, C. Zhang, and L. Liu, "Design and Control of a Single-MotorActuated Robotic Fish Capable of Fast Swimming and
Maneuverability," IEEE/ASME Transactions on Mechatronics, vol. 21, no. 3, 2016, pp. 1711-1719.

[16] M. Hassanalian, H. Abdelmoula, S. Mohammadi, S. Bakhtiyarov, J. Goerlich, and U. Javed, "Aquatic animal colors and skin temperature: Biology's selection for reducing oceanic dolphin's skin friction drag," Journal of Thermal Biology, vol. 84, 2019, pp. 292-310.

[17] Y. Zhang, S. Wang, X. Wang, and Y. Geng, "Design and Control of Bionic Manta Ray Robot With Flexible Pectoral Fin," IEEE 14th International Conference on Control and Automation, pp. 1034- 1039.

[18] P. V. y Alvarado, S. Chin, W. Larson, A. Mazumdar, and K. Youcef Toumi, "A soft body under-actuated approach to multi degree of freedom biomimetic robots: A stingray example," in Biomedical Robotics and Biomechatronics (BioRob), 2010 3rd IEEE RAS and EMBS International Conference on, pp. 473-478.

[19] P. V. y Alvarado, "Hydrodynamic performance of a soft body underactuated batoid robot," in Robotics and Biomimetics (ROBIO), 2011 IEEE International Conference on, pp. 1712-1717.

[20] A. Cloitre, V. Subramaniam, N. Patrikalakis, and P. V. y Alvarado, "Design and control of a field deployable batoid robot," in Biomedical Robotics and Biomechatronics (BioRob), 2012 4th IEEE RAS \& EMBS International Conference on, pp. 707-712.

[21] K. S. Sekar, M. Triantafyllou, and P. y Valdivia y Alvarado, "Flapping actuator inspired by lepidotrichia of ray-finned fishes," in Intelligent Robots and Systems (IROS 2014), 2014 IEEE/RSJ International Conference on, pp. 1120-1126.

[22] C. M. Chew, Q. Y. Lim, and K. S. Yeo, "Development of propulsion mechanism for Robot Manta Ray," in Robotics and Biomimetics (ROBIO), 2015 IEEE International Conference on, pp. 1918-1923.

[23] T. V. Truong, V. K. Viswanathan, V. S. Joseph and P. V. y. Alvarado, "Design and Characterization of a Fully Autonomous Under-actuated Soft Batoid-like Robot," 2019 IEEE/RSJ International Conference on Intelligent Robots and Systems (IROS), 2019, pp. 5826-5831. 